\title{
Polimorfismo Pro72Arg del gen TP53 se asocia a enfermedad coronaria en individuos Chilenos
}

\author{
José Caamaño L1, ${ }^{1}$, Nicolás Saavedra ${ }^{1}$, Priscilla C. Jaramillo ${ }^{1}$, Cecilia Lanas ${ }^{2}$, Fernando Lanas ${ }^{2}$, Luis A. Salazar ${ }^{1}$ \\ ${ }^{1}$ Laboratorio de Biología Molecular \& Farmacogenética, Departamento de Ciencias Básicas, Facultad de \\ Medicina, Universidad de La Frontera \& ${ }^{2}$ Departamento de Medicina Interna, Facultad de Medicina, Universidad \\ de La Frontera, Temuco, Chile. \\ Trabajo financiado por Convenio de Desempeño-l y II-2008 (LS), Dirección de Investigación y Desarrollo, Universidad \\ de La Frontera, y MIDEPLAN
}

\section{Resumen}

Introducción: Diferentes genes han sido implicados en la etiología de la enfermedad arterial coronaria, entre ellos, el gen TP53. Recientemente, el polimorfismo en el codon 72 (Pro72Arg, rs1042522) del gen TP53 fue señalado como factor de riesgo para enfermedad arterial coronaria. Sin embargo, otros autores no han confirmado esta observación. Así, en el presente estudio investigamos la posible asociación entre esta variante genética y la presencia de enfermedad arterial coronaria en individuos chilenos.

Métodos: Se analizaron 209 pacientes, no relacionados, con diagnóstico de enfermedad arterial coronaria confirmada por angiografía (estenosis > 70\%), 33 - 74 años y 216 individuos controles (30-68 años). Las concentraciones séricas de glucosa, acido úrico, triglicéridos, colesterol total y colesterol HDL fueron determinados por métodos enzimático-colorimétricos. El polimorfismo Pro72Arg del gen TP53 fue identificado mediante la técnica de reacción en cadena de polimerasa seguida de restricción enzimática (PCR-RFLP).

Resultados: La distribución de genotipos para la mutación Pro72Arg del gen TP53 en pacientes y controles fue significativamente diferente $(P=0.003)$. Adicionalmente, la frecuencia relativa de alelos fue también diferente $(P=0.003)$. La OR para enfermedad coronaria relacionada al alelo $72 \mathrm{Arg}$ fue 2.0 (I.C.95\%=1.33-2.90), confirmando la presencia de asociación. Por otro lado, no encontramos asociación entre los factores de riesgo tradicionales para enfermedad coronaria y los diferentes genotipos del polimorfismo Pro72Arg.

Conclusión: Nuestro estudio muestra una interesante asociación entre enfermedad coronaria y el polimorfismo Pro72Arg del gen TP53 en individuos chilenos, sugiriendo que esta mutación podría ser útil como marcador genético de esta patología. Sin embargo, esta observación necesita ser reconfirmada con un estudio poblacional.

Palabras clave: Enfermedad arterial coronaria; Polimorfismo genético; TP53; Factores de riesgo

TP53 codon 72 polymorphism is associated with coronary artery disease in Chilean subjects

Background: Different genes have been implicated in the aetiology of coronary artery disease, among these, the TP53 gene. Recently, the codon 72 polymorphism (Pro72Arg, rs1042522) of TP53 gene was indicated as a risk factor for coronary artery disease (CAD). However, other authors do not confirm this observation. Thus, in the

Correspondencia: Dr. Luis Antonio Salazar

Departamento de Ciencias Básicas, Facultad de Medicina,

Universidad de La Frontera.

Av. Francisco Salazar 01145, Casilla 54-D, Temuco

Fono: (45) 592895 - Fax: (45) 592832.

Correo Electrónico: Isalazar@ufro.cl 
present study we investigated the possible association between this genetic variant and the presence of $C A D$ in Chilean subjects.

Methods: 209 unrelated patients with diagnosis of CAD confirmed by angiography (33-74 years old) and 216 healthy controls (30 - 68 years old) were included in this study. The Pro72Arg polymorphism of the TP53 gene was evaluated by PCR-RFLP.

Results: The genotype distribution for Pro72Arg variant of TP53 gene in CAD patients (PP: 6.2\%, PR: 29.2\%, $R R: 64.6 \%)$ and controls (PP: $8.3 \%, P R: 43.5 \%, R R: 48.1 \%)$ was significantly different $(P=0.003)$. Similarly, the allelic frequency was also different $(P=0.003)$. The OR for CAD related to $72 \mathrm{Arg}$ allele was $2.0(95 \% \mathrm{C} . \mathrm{I} .=1.33-2.90)$.

Conclusion: These findings suggest that the Pro72Arg polymorphism of the TP53 gene is associated with CAD in study Chilean individuals.

Key words: cardiovascular risk factors, Genetic polimorfism, TP53

Recibido el 16 de diciembre de 2008. Aceptado el 10 de mayo de 2009

\section{Introducción}

La enfermedad arterial coronaria (EAC) es una enfermedad poligénica cuyas bases moleculares se encuentran bajo investigación. Diferentes genes han sido implicados en la etiología de EAC1, entre ellos, el gen TP532. La principal función del gen TP53 es proteger a las células frente a la transformación maligna, prevenir la proliferación de células conteniendo ADN dañado, y si la reparación no es posible, activar el mecanismo por el cual los tejidos remueven las células no deseadas, viejas o dañadas; la vía de la apoptosis ${ }^{3}$. Este mecanismo ha sido relacionado a la etiología de la lesión aterosclerótica4; sin embargo, el rol de TP53 no ha sido completamente comprendido.

El gen TP53 funciona como un antioncogen, asociado con la sobre-regulación de apoptosis2. TP53 wild-type se acumula en el tejido aterosclerótico y puede mediar la apoptosis de las células vasculares $^{5}$. Además, se ha sugerido que TP53 regula la división celular y la apoptosis en la placa aterosclerótica dependiendo del tipo celular y nivel de expresión de TP53 inducido por el daño al ADN 6, 7 . Mutaciones que afecten al gen TP53 pueden inducir disfunciones de la proteína p53 e inhibir apoptosis, además se ha sugerido que la perdida de actividad del gen TP53 puede jugar un papel relevante en la patogénesis de la aterosclerosis 5,8 .
Rev Chil Cardiol 2009; 28: 151-157

Un polimorfismo común en el gen TP53, Pro72Arg (rs1042522), resulta en la ocurrencia de arginina (Arg) o prolina (Pro) en el codon 72 en la secuencia aminoacídica de la proteína. Este polimorfismo ha sido asociado con varios tipos de cáncer9-13, lupus eritematoso sistémico14 y endometriosis ${ }^{15}$. Recientemente, la posible asociación con enfermedad cardiovascular fue investigada16-18. Sin embargo, los resultados son contradictorios.

En Chile, las enfermedades cardiovasculares son la principal causa de muerte ${ }^{19}$. Sin embargo, la información en relación a las bases genéticas de EAC en nuestro país es insuficiente20-22. Así, el objetivo del presente estudio fue evaluar la posible asociación entre el polimorfismo Pro72Arg del gen TP53 y EAC en individuos chilenos.

\section{Material y Métodos}

\section{Sujetos de estudio}

El polimorfismo Pro72Arg se estudió en 209 pacientes no relacionados, los que conformaron el grupo de casos, cuyas edades fluctuaron entre los 33 y 74 años, ingresados al servicio de Cardiología del Hospital Dr. Hernán Henríquez de Temuco, con diagnóstico de enfermedad arterial coronaria demostrada por angiografía (estenosis de la arteria coronaria 
$>70 \%$ ). El grupo control lo constituyeron 216 individuos no relacionados con edades entre los 30 y 68 años, todos procedentes de la ciudad de Temuco, sin enfermedad coronaria demostrada por la ausencia de angina y electrocardiograma sin evidencias de isquemia o infarto.

Se empleó un cuestionario estructurado para identificar los controles libres de enfermedad y para excluir sujetos que fueran sospechosos de tener alguna forma de enfermedad vascular. Aquellos individuos con historia familiar de EAC fueron excluidos del estudio. Datos demográficos e historia de hipertensión, diabetes mellitus, consumo de tabaco e hipercolesterolemia fueron evaluados en cada sujeto. No hubo preselección de concentración de lípidos sanguíneos en ambos grupos. Sujetos con una historia de diabetes o que tuvieran glicemia basal $\geq 126$ $\mathrm{mg} / \mathrm{dL}$ fueron definidos como diabéticos. El protocolo de estudio fue aprobado por el comité de ética de nuestra Universidad, además todos los sujetos firmaron un consentimiento informado.

\section{Determinaciones bioquímicas}

Para las determinaciones de los parámetros bioquímicos se obtuvo una muestra de sangre colectada en tubos sin anticoagulante luego de un ayuno de 12 horas. Los niveles séricos de glucosa, acido úrico, triglicéridos, colesterol total y colesterol HDL fueron determinados mediante métodos enzimáticocolorimétricos $^{20}$. El colesterol de las lipoproteínas de baja densidad (LDL-C) fue calculado usando la formula de Friedewald cuando la concentración de triglicéridos no excedió los $400 \mathrm{mg} / \mathrm{dL}$.

\section{Genotipificación de la variante Pro72Arg del gen TP53}

El ADN genómico fue extraído a partir de leucocitos sanguíneos empleando el método de precipitación salina optimizado por Salazar et al.23. El polimorfismo Pro72Arg del gen TP53 fue detectado usando reacción en cadena de la polimerasa (PCR) seguido de restricción enzimática de acuerdo a las condiciones descritas por Smith et al. ${ }^{16}$.

\section{Análisis estadístico}

El análisis estadístico fue realizado utilizando el programa Sigma Stat para Windows, versión. 2.0 (Jandel Sci., San Rafael, CA, USA). Los datos son presentados como media $\pm D E$. Diferencias entre los promedios de las variables continuas fueron evaluadas a través del test $t$ de Student o ANOVA. Las frecuencias alélicas y la distribución de genotipos fueron estimadas por conteo de genes. Diferencias entre variables no continuas y el equilibrio de HardyWeinberg fueron calculadas mediante análisis de Chi-cuadrado $\left(\mathrm{X}^{2}\right)$. La odds ratio (OR) y su intervalo de confianza (IC) de $95 \%$ asociado al alelo mutado $72 \mathrm{R}$ fueron también calculados. El nivel de significancia estadística fue $\mathrm{P}<0.05$.

\section{Resultados}

\section{Características de los sujetos}

Las características clínicas, antropométricas $y$ de laboratorio de los sujetos en estudio son mostradas en la Tabla 1. Los individuos con EAC presentan valores elevados de índice de masa corporal (IMC) y una mayor prevalencia de factores de riesgo tradicionales para EAC, incluyendo consumo de tabaco, diabetes, hipertensión, hipercolesterolemia e historia familiar de EAC $(P<0.001)$. Las concentraciones basales de colesterol total, colesterol LDL, triglicéridos, glucosa y acido úrico fueron más altas en los pacientes con EAC que en los controles $(P<0.001)$. Además, los individuos con EAC presentaron niveles más bajos de HDL-C $(P<0.001)$. Similarmente, se advirtió una diferencia significativa en los valores promedio de presión arterial sistólica $(P<0.001)$ y diastólica $(P=0.020)$ entre los grupos EAC y controles.

\section{Frecuencia de Alelos}

La distribución de genotipos y la frecuencia relativa de alelos del polimorfismo Pro72Arg del gen TP53 en los pacientes con EAC y controles son mostradas en la Tabla 2. La distribución de genotipos fue la esperada para el equilibrio de Hardy-Weinberg para los sujetos con EAC y controles $\left(E A C, X^{2}=2.743\right.$, P=NS y Controles, $X^{2}=0.255, P=N S$ ); y similar a datos previos observados en una muestra de población chilena adulta14,15. La distribución de genotipos para el polimorfismo Pro72Arg en pacientes con EAC y controles fue significativamente diferente $(P=0.03)$. 
Además, la frecuencia relativa de alelos fue diferente en ambos grupos $(P=0.003)$. La OR para EAC relacionada al genotipo homocigoto RR fue 2.00 (I.C.95\%, 1.33-2.90) confirmando la presencia de asociación.

Tabla 1: Características clínicas y demográficas de los sujetos con enfermedad arterial coronaria (casos) y controles.

\begin{tabular}{lccc}
\hline & $\begin{array}{c}\text { Casos } \\
(\mathbf{2 0 9 )}\end{array}$ & $\begin{array}{c}\text { Controles } \\
(\mathbf{2 1 6})\end{array}$ & $\mathbf{P}^{*}$ \\
\hline Edad, años & $62 \pm 10$ & $42 \pm 8$ & $<0.001$ \\
Hombres, \% & 64 & 56 & 0.084 \\
Diabetes, \% & 32 & 5 & $<0.001$ \\
PAS, mm Hg & $140 \pm 26$ & $125 \pm 23$ & $<0.001$ \\
PAD, mm Hg & $80 \pm 18$ & $75 \pm 9$ & 0.020 \\
IMC, kg/m2 & $28.2 \pm 4.5$ & $25.8 \pm 4.5$ & $<0.001$ \\
Fumadores, \% & 64 & 41 & $<0.001$ \\
Hipercolesterolemia, \% & 80 & 20 & $<0.001$ \\
Hipertensión, \% & 79 & 26 & $<0.001$ \\
Angina, \% & 77 & 0 & $<0.001$ \\
IAM, \% & 66 & 0 & $<0.001$ \\
Historia Familiar de EAC, \% & 32 & 0 & $<0.001$ \\
Número de vasos obstruídos, \% & & & \\
$\quad 1$ & $28 \%$ & 0 & -- \\
$\quad 2$ & $24 \%$ & 0 & -- \\
$\quad 3$ & $48 \%$ & 0 & --- \\
Colesterol total, mg/dL & $214 \pm 51$ & $175 \pm 33$ & $<0.001$ \\
LDL - C, mg/dL & $127 \pm 42$ & $95 \pm 27$ & $<0.001$ \\
HDL - C, mg/dL & $32 \pm 8$ & $49 \pm 12$ & $<0.001$ \\
Triglicéridos, mg/dL & $182 \pm 193$ & $114 \pm 86$ & $<0.001$ \\
Glucosa, mg/dL & $113 \pm 42$ & $94 \pm 37$ & $<0.001$ \\
Acido Úrico, mg/dL & $5.6 \pm 1.6$ & $4.5 \pm 1.5$ & $<0.001$ \\
\hline
\end{tabular}

Número de individuos en paréntesis; IAM; infarto agudo al miocardio; IMC, índice de masa corporal; EAC, enfermedad arterial coronaria; PAS, presión arterial sistólica; PAD, presión arterial diastólica; HDL-C, Colesterol de las HDL; LDL-C, Colesterol de las LDL. *Test $t$ de Student o Chi-cuadrado.

Tabla 2: Distribución de genotipos y frecuencia relativa de alelos del polimorfismo Pro72Arg del gen TP53 en individuos chilenos con enfermedad arterial coronaria (casos) y controles.

\begin{tabular}{lcccccc}
\hline & \multicolumn{3}{c}{ Genotipo } & $\mathrm{P}$ & Alelo \\
\cline { 2 - 6 } & $\mathrm{PP}$ & $\mathrm{PR}$ & $\mathrm{RR}$ & 0.208 & 0.792 \\
\hline Casos & $6.2 \%$ & $29.2 \%$ & $64.6 \%$ & & \\
$(209)$ & $(13)$ & $(61)$ & $(135)$ & 0.301 & 0.699 \\
Controles & $8.3 \%$ & $43.5 \%$ & $48.1 \%$ & & \\
$(216)$ & $(18)$ & $(94)$ & $(104)$ & $\mathrm{X}^{2}=9.14 ; 1 \mathrm{gl} ; \mathrm{P}=0.003$ \\
& $\mathrm{X}^{2}=11.74 ; 2 \mathrm{gl} ; \mathrm{P}=0.003$ &
\end{tabular}

Número de individuos en paréntesis; gl, grados de libertad. 


\section{Discusión}

La enfermedad arterial coronaria es una compleja enfermedad genética y numerosos genes han sido relacionados con su etiología1, 24. Entre esos genes, el gen supresor de tumores TP5325-27. Un polimorfismo común en TP53, Pro72Arg, ha sido objeto de análisis en EAC; sin embargo, los resultados son discordantes16-18. En el presente estudio, hemos investigado la posible asociación entre el polimorfismo Pro72Arg y enfermedad arterial coronaria en individuos chilenos.

El análisis de los parámetros clínicos y de laboratorio en ambos grupos estudiados, muestra que las concentraciones séricas de colesterol total, colesterol LDL, triglicéridos, glucosa y ácido úrico fueron más altas en los sujetos con EAC. Además, las concentraciones de HDL-C fueron más bajas en este grupo; esos resultados confirman la conocida asociación entre esos factores de riesgo tradicionales y EAC28.

Nuestros hallazgos muestran también una significativa asociación entre EAC y la presencia del alelo R (variante Arg72) del polimorfismo Pro72Arg del gen TP53 (OR=2.00, I.C. 95\% 1.33-2.90). La frecuencia del alelo $R$ fue más alta en los sujetos con EAC que en los controles ( 0.79 vs. $0.69, P=0.003$ ). Similarmente, Kojima et al.29, evaluaron el polimorfismo Pro72Arg del gen TP53 en 66 individuos con angioplastía primaria con balón, encontrando una significativa asociación entre el genotipo homocigoto Arg/Arg y el estrechamiento de la arteria coronaria. Además, Bonafé et al.30, en un elegante estudio, mostraron que los individuos portadores de la variante Arg exhiben niveles aumentados de CK-MB y troponina I, ambos marcadores bioquímicos de injuria miocárdica. Adicionalmente, Bonafé et al. 31 revelaron que la variante $72 \mathrm{Arg}$ se asocia con restenosis después de angioplastía. Estos antecedentes sugieren que el polimorfismo del codon 72 del gen TP53 puede contribuir a una variabilidad genéticamente determinada en aterosclerosis.

Por otra parte, Manfredi et al. 18 no encontraron ninguna asociación entre el polimorfismo Pro72Arg y la ocurrencia de EAC en individuos italianos.
Adicionalmente, Smith et al.16 analizando población Brasileña, no lograron establecer una asociación entre este polimorfismo y enfermedad cardiovascular $(P=0.071)$. No obstante, esos autores detectaron una asociación entre el alelo 72Arg y bajos niveles de colesterol HDL. Análogamente, Alkhalaf et al. 17 no encontraron asociación entre el polimorfismo del codon 72 del gen TP53 y EAC o diabetes en individuos de Kuwait.

En relación a los posibles mecanismos que puedan explicar nuestros descubrimientos, es importante mencionar que el polimorfismo Pro72Arg afecta al dominio rico en prolina de p53, secuencia necesaria para la transmisión de señales antiproliferativas 32 . Se ha sugerido además, que los dos alelos pueden producir proteínas funcionalmente distintas, con diferencias en su capacidad de activar la expresión de genes 33 . Dumont et al. 34 establecieron que la variante 72 Arg es más eficiente en inducir apoptosis, probablemente a causa de una mejor interacción con MDM2, facilitando la exportación nuclear y localización mitocondrial de p53. Además, el polimorfismo Pro72Arg ha sido correlacionado con la variación inter-individual en la respuesta apoptótica.

En la placa aterosclerótica, especies reactivas de oxígeno son responsables del daño al ADN7, y es sabido que una sola ruptura en una molécula de doble hebra de ADN puede ser suficiente para promover un aumento en los niveles de p5335. La apoptosis en las células endotelilales ha sido relacionada a inestabilidad de la placa y formación de trombos ${ }^{36}$. Un disturbio en la respuesta apoptótica puede llevar a acumulación de células de la íntima que progresen a aterogénesis ${ }^{4}$. La consecuencia funcional del polimorfismo Pro72Arg ha sido relacionada a la inhibición la función de p73, un miembro de la familia de p53 de factores de transcripción nuclear, implicados en la supresión de tumores 37 , 38. El alelo Arg es más eficiente para unirse a p73; bloquear su acción y facilitar la proliferación de células vasculares lisas 37,39 . De acuerdo a esta evidencia, es probable que los individuos con EAC portadores de la variante Arg del polimorfismo Pro72Arg del gen TP53, sean más susceptibles a 
sufrir desregulación de la apoptosis durante la progresión aterosclerótica, y consecuentemente, promover el desarrollo de enfermedad cardiovascular. Esta idea es concordante con el comportamiento del polimorfismo Pro72Arg en diferentes tipos de cáncer. Storey et al. 40 establecieron que los sujetos homocigotos para la variante Arg72 son siete veces más susceptibles a tumorogénesis asociada al virus papiloma que los heterocigotos.

En resumen, nuestro estudio muestra una interesante asociación entre EAC y el polimorfismo del codon 72 del gen TP53 en individuos chilenos y sugiere que esta variante alélica podría ser un marcador genético para EAC en nuestro país. Sin embargo, esta observación necesita ser confirmada en forma independiente por otros grupos a nivel nacional. Además, es importante considerar para futuros estudios, una selección más rigurosa de los individuos controles. Idealmente, deberían incluirse individuos que no presenten lesiones, a los cuales se les realizó coronariografía por otros motivos (por ejemplo, pases operatorios), y con edades similares a los casos.

\section{Referencias}

1. WANG Q. Molecular genetics of coronary artery disease. Curr Opin Cardiol 2005; 20: 182-8.

2. GENG YJ. Molecular signal transduction in vascular cell apoptosis. Cell Res 2001; 11: 253-64.

3. BOSE I, GHOSH B. The p53-MDM2 network: from oscillations to apoptosis. J Biosci 2007; 32: 991-7.

4. GENG YJ, LIBBY P. Progression of atheroma: a struggle between death and procreation. Arterioscler Thromb Vasc Biol 2002; 22: 1370-80.

5. AOKI M, MORISHITA R, MATSUSHITA H, NAKANO N, HAYASHI S, TOMITA N, et al. Serum deprivation-induced apoptosis accompanied by up-regulation of p53 and bax in human aortic vascular smooth muscle cells. eart Vessels1997; 12: 71-5.

6. MERCER J, BENNETT M. The role of p53 in atherosclerosis. Cell Cycle 2006; 5: 1907-9.

7. MERCER J, MAHMOUDI M, BENNETT M. DNA damage, p53, apoptosis and vascular disease. Mutat Res 2007; 621: 75-86.

8. GUEVARA NV, KIM HS, ANTONOVA EI, CHAN L. The absence of $p 53$ accelerates atherosclerosis by increasing cell proliferation in vivo. Nat Med 1999; 5: 335-9.

9. PINTO GR, YOSHIOKA FK, SILVA RL, CLARA CA, SANTOS MJ, ALMEIDA JR, et al. Prognostic value of TP53 Pro47Ser and Arg72Pro single nucleotide polymorphisms and the susceptibility to gliomas in individuals from Southeast Brazil. Genet Mol Res 2008; 7: 207-16.

10. COSTA S, PINTO D, PEREIRA D, RODRIGUES $H$, CAMESELLE-TEIJEIRO J, MEDEIROS R, et al. Importance of TP53 codon 72 and intron 3 duplication 16bp polymorphisms in prediction of susceptibility on breast cancer. BMC Cancer 2008; 8: 32.

11. ZHU ZZ, WANG AZ, JIA HR, et al. Association of the TP53 codon 72 polymorphism with colorectal cancer in a Chinese population. Jpn J Clin Oncol 2007; 37(5):385-90.
12. OJEDA JM, AMPUERO S, ROJAS P, et al. p53 codon 72 polymorphism and risk of cervical cancer. Biol Res 2003; 36: 279-83.

13. IRARRÁZABAL CE, ROJAS C, ARACENA R, MÁRQUEZ C, GIL L. Chilean pilot study on the risk of lung cancer associated with codon 72 polymorphism in the gene of protein $\mathrm{p} 53$. Toxicol Lett 2003; 144: 69-76.

14. PIOTROWSKI P, LIANERI M, MOSTOWSKA M, WUDARSKI $\mathrm{M}$, CHWALINSKA-SADOWSKA $\mathrm{H}$, et al. Contribution of polymorphism in codon 72 of p53 gene to systemic lupus erythematosus in Poland. Lupus 2008; 17: 148-51.

15. HSIEH YY, LIN CS. P53 codon 11, 72, and 248 gene polymorphisms in endometriosis. Int J Biol Sci 2006; 2: 188-93

16. SMITH MA, SILVA MD, CENDOROGLO MS, RAMOS LR, ARAUJO LM, LABIO RW, et al. TP53 codon 72 polymorphism as a risk factor for cardiovascular disease in a Brazilian population. Braz J Med Biol Res 2007; 40: 1465-72.

17. ALKHALAF M, AL-BUSTAN S, HAMODA H, ABDELLA N. Polymorphism of p53 gene codon 72 in Kuwaiti with coronary artery disease and diabetes. Int J Cardiol 2007; 115: 1-6.

18. MANFREDI S, MASETTI S, BOTTO N, COLOMBO MG, TERRAZZI M, VASSALLE C, et al. P53 codon 72 polymorphism in coronary artery disease: no evidence for association with increased risk or micronucleus frequency. Environ Mol Mutagen. 2002; 40: 110-5.

19. LANAS F, AVEZUM A, BAUTISTA LE, DIAZ R, LUNA M, ISLAM S, et al. Risk factors for acute myocardial infarction in Latin America: the INTERHEART Latin American study. Circulation 2007; 115: 1067-74.

20. JARAMILLO PC, MUÑOZ A, LANAS C, LANAS F, SALAZAR LA. Endothelial nitric oxide synthase G894T gene polymorphism in Chilean subjects with coronary artery disease and controls. Clin Chim Acta 2006; 371: 102-6.

21. HERRERA L, JARAMILLO PC, LANAS F, SALAZAR LA. Polymorphism C373G of the PECAM - 1 gene in Chilean 
subjects with coronary disease and controls. Int J Morphol 2007; 25: 443-448.

22. JARAMILLO PC, LANAS C, LANAS F, SALAZAR LA. -786T >C polymorphism of the endothelial nitric oxide synthase gene in Chilean subjects with coronary artery disease and controls. Clin Chim Acta 2008; 387: 105-108.

23. SALAZAR LA, HIRATA M, CAVALLI SA, MACHADO MO, HIRATA RD. Optimized procedure DNA isolation from fresh and cryopreserved clotted human blood useful in clinical molecular testing. Clin Chem 1998; 44: 1748-50.

24. MAYER B, ERDMANN J, SCHUNKERT H. Genetics and heritability of coronary artery disease and myocardial infarction. Clin Res Cardiol 2007; 96: 1-7.

25. AOKI M, MORISHITA R, MATSUSHITA H, HAYASHI S, NAKAGAMI H, YAMAMOTO $\mathrm{K}$, et al. Inhibition of the $\mathrm{p} 53$ tumor suppressor gene results in growth of human aortic vascular smooth muscle cells. Potential role of p53 in regulation of vascular smooth muscle cell growth. Hypertension 1999; 34: 192-200.

26. TABAS I. p53 and atherosclerosis. Circ Res 2001; 88: 747-9

27. WANG XL, WANG J, WILCKEN DE. Interactive effect of the p53 gene and cigarette smoking on coronary artery disease. Cardiovasc Res 1997; 35: 250-5.

28. Executive summary of the third report of the National Cholesterol Education Program (NCEP) Expert panel on detection, evaluation, and treatment of high blood cholesterol in adults (Adults treatment Panel III). J Am Med Assoc 2001; 285: 2486-97.

29. KOJIMA S, GOTO Y, NONOGI H, HORIE H, KINOSHITA M, IWAI N. Role of a p53 polymorphism in luminal narrowing after balloon coronary angioplasty. Atherosclerosis 2000; 151: 585-6.

30. BONAFÉ M, SALVIOLI S, BARBI C, TRAPASSI C, TOCCO F, STORCI G, et al. The different apoptotic potential of the p53 codon 72 alleles increases with age and modulates in vivo ischaemia-induced cell death. Cell Death Differ 2004; 11: 962-73.

31. BONAFÉ M, OLIVIERI F, MARI D, BAGGIO G, MATTACE R, BERARDELLI M, et al. P53 codon 72 polymorphism and longevity: additional data on centenarians from continental Italy and Sardinia. Am J Hum Genet 1999; 65: 1782-5.

32. WALKER KK, LEVINE AJ. Identification of a novel p53 functional domain that is necessary for efficient growth suppression. Proc Natl Acad Sci USA 1996; 93: 15335-40.

33. THOMAS M, KALITA A, LABRECQUE S, PIM D, BANKS L, MATLASHEWSKI G. Two polymorphic variants of wild-type p53 differ biochemically and biologically. Mol Cell Biol 1999; 19: 1092-100.

34. DUMONT P, LEU JI, DELLA PIETRA AC 3RD, GEORGE DL, MURPHY M. The codon 72 polymorphic variants of $p 53$ have markedly different apoptotic potential. Nat Genet 2003; 33: 357-65.

35. VOGELSTEIN B, LANE D, LEVINE AJ. Surfing the p53 network. Nature 2000; 408: 307-10.

36. KAVURMA MM, BHINDI R, LOWE HC, CHESTERMAN C, KHACHIGIAN LM. Vessel wall apoptosis and atherosclerotic plaque instability. J Thromb Haemost 2005; 3: 465-72.

37. MARABESE M, VIKHANSKAYA F, BROGGINI M. p73: a chiaroscuro gene in cancer. Eur J Cancer 2007; 43: 1361-72.

38. OZAKI T, NAKAGAWARA A. p73, a sophisticated p53 family member in the cancer world. Cancer Sci 2005; 96: 729-37.

39. KOJIMA S, IWAI N, TAGO N, ONO K, OHMI K, TSUJIMOTO $\mathrm{G}$, et al. p53Arg72Pro polymorphism of tumour suppressor protein is associated with luminal narrowing after coronary stent placement. Heart 2004; 90: 1069-70.

40. STOREY A, THOMAS M, KALITA A, HARWOOD C, GARDIOL $D$, MANTOVANI $F$, et al. Role of a p53 polymorphism in the development of human papillomavirus-associated cancer. Nature 1998; 393: 229-34. 